\title{
Sang et statut des homicides chez les Yanomami centraux (Venezuela)
}

Blood and the status of homicide among the central Yanomami (Venezuela)

Jacques Lizot

\section{OpenEdition}

\section{Journals}

Édition électronique

URL : http://journals.openedition.org/span/1504

DOI : $10.4000 /$ span. 1504

ISSN : 2268-1558

Éditeur

École pratique des hautes études. Sciences humaines

\section{Édition imprimée}

Date de publication : 1 août 1996

Pagination : 105-126

ISSN : 0294-7080

\section{Référence électronique}

Jacques Lizot, «Sang et statut des homicides chez les Yanomami centraux (Venezuela) », Systèmes de pensée en Afrique noire [En ligne], 14 | 1996, mis en ligne le 17 avril 2014, consulté le 10 octobre 2020. URL : http://journals.openedition.org/span/1504 ; DOI : https://doi.org/10.4000/span.1504 


\title{
SANG ET STATUT DES HOMICIDES CHEZ LES YANOMAMI CENTRAUX (Venezuela)
}

\author{
par
}

\section{Jacques Lizot}

Dans de nombreuses sociétés le contact du sang menstruel et le statut des meurtriers sont l'objet de prescriptions et de prohibitions qui s'inscrivent dans des cérémonies plus ou moins complexes et rigoureuses. C'est le cas des Yanomami, au Venezuela. Ces Indiens appellent du même nom, unokai, l'état d'une femme qui a ses premières règles et celui d'un homicide. Dans l'un et l'autre cas le déroulement du rituel est à peu près le même et celui-ci s'achève par une cérémonie identique, le paushimou'. C'est de ce rituel que nous allons d'abord parler, et nous nous demanderons pourquoi la première menstruation d'une femme et la situation rituelle d'un meurtrier sont pensées et traitées de manière identique par les Indiens. Qu'y a-t-il de commun dans les deux cas ? Puis, dépassant ce point de vue initial, nous déterminerons le statut social du guerrier.

D’une manière générale le sang des animaux et celui des blessés n'est pas dangereux, mais on ne mange la chair des animaux (poissons

1 paushimou c'est, dans la langue courante, se parer (de plumes, de peaux d'oiseaux, de colliers ou de fleurs).

Destins de meurtriers

Systèmes de pensée en Afrique noire, 14, 1996 
inclus) qu'après qu'il ait été totalement éliminé par une cuisson prolongée. Il ne faut jamais consommer une chair sanguinolente, sous peine de se métamorphoser en bête sauvage, ou de voir son corps se couvrir de plaies inguérissables (washë) ${ }^{2}$; ingérer une viande qui porte encore des traces de sang, c'est se comporter comme un prédateur qui mange de la viande crue : c'est être cannibale. Les animaux domestiques n'échappent pas à ce danger et j'ai entendu raconter l'histoire d'un chien qui s'était transformé en poisson pour en avoir mangé un cru. Le rouge est la couleur qui symbolise le sang et le roucou, un colorant rouge vif, doit être, pour être bon, rouge comme un caillot de tapir.

Nous venons de situer approximativement la signification du sang dans la culture yanomami ${ }^{3}$. Examinons maintenant le cas particulier du rituel unokaimou, en commençant par les femmes qui ont leurs premières règles. Dès qu'une jeune fille se rend compte qu'elle a ses règles, elle en informe sa mère ${ }^{4}$. Si par hasard elle se trouve à ce moment dans la forêt, elle doit cesser de regarder autour d'elle, sous peine de susciter l'agressivité des guêpes. La mère (ou une tante ou, à défaut, une sæur aînée) va aussitôt cueillir des branches de * feuilles à menstrues " (Sorocea guyanensis). Cet arbuste, en période de floraison, donne de modestes fleurs dont le calice, d'une seule pièce, est rouge-vif. Lorsqu'elle est de retour, la mère dresse les branchages dans la partie basse de l'auvent communautaire, aménageant ainsi un enclos plus ou moins rectangulaire de manière à soustraire sa fille aux regards. Là, recluse derrière la frêle paroi de feuillages, la jeune fille s'allonge dans un hamac en écorce tendu entre deux poteaux de soutènement situés en arrière du toit. Un feu brûle près d'elle. Elle a retiré la torsade de coton qu'elle portait autour de la poitrine et son

${ }^{2}$ La description des symptômes par les Yanomami fait penser à la leishmaniose. Nous verrons que ce mal, pensé par les Indiens comme une pourriture des chairs, guette le meurtrier qui a * mangé * sa victime. Ces mêmes plaies pourraient couvrir le corps d'un homicide si celui-ci venait à fouler le sang de sa victime.

3 On écrit Yanomami lorsqu'on veut désigner l'ethnie, Yanomamë et Yanomami pour désigner deux des quatre sous-groupes.

${ }^{4}$ On dit que les écureuils kario (Sciurus granatensis) ont leurs règles en même temps que les filles. 
tablier de coton. Le tabac lui est permis, à condition qu'il soit presque sans goût, lavé à force d'avoir été sucé. Elle a aussi retiré ses bâtonnets d'oreille. Le contact direct avec l'eau lui est interdit et, pour boire, elle se sert d'un tube creux qu'elle enfonce dans sa bouche en arrière de la dentition: ses dents se déchausseraient si elles entraient directement en contact avec l'eau. Elle possède un petit morceau de bois épointé pour se gratter : si, par mégarde, elle utilisait les ongles, ses poils et ses cheveux tomberaient, sa peau se couvrirait de plaies. Pour attiser le feu, elle prend un éventail au lieu de souffler. Elle ne peut parler qu'à voix basse, même lorsqu'une de ses compagnes (seule admise à venir auprès d'elle et à la "voir ») vient se glisser à travers le feuillage pour lui faire la conversation ; l'éclat de leurs voix ne doit pas être perçu de l'extérieur. Son régime alimentaire est sévèrement restreint, le bouilli et la viande en général lui sont interdits, ainsi que les bananes mûres et la canne à sucre. Elle n'a droit quotidiennement qu'à quelques bananes-plantains vertes rôties sur braise, ou à du taro cuit de la même manière ; il lui est interdit de saisir avec la main cette maigre pitance qu'elle manipule avec une baguette piquée dedans. Elle a aussi la possibilité de suçoter de temps à autre la carapace d'un crabe. Elle ne doit pas manger les fruits du palmier rasha ${ }^{5}$, rouges comme le sang, sinon une épine de ce palmier viendrait se ficher dans l'ceil de son mari. Elle urine au centre de son abri et défèque dans des feuilles dont elle fait un paquet que sa mère ira jeter à l'extérieur.

D'une recluse on dit qu'elle a «valeur d'humidité " s'il pleut beaucoup pendant son isolement. Les jeunes filles friandes de yupu una, un condiment liquide préparé avec les cendres d'une écorce, tombent toutes dans cette catégorie. Les Indiens disent que chaque lunaison est accompagnée, comme les femmes qui ont leurs premières règles, de beau ou de mauvais temps, et les femmes associent la

5 Les rasha sont des fruits de palmiers cultivés (Guilielma gasipaes) qui viennent à maturité entre décembre et février, pendant la saison sèche. Ils sont très appréciés des Indiens. Il existe différentes variétés et les fruits vont du vert jaunâtre au rouge vif. La variété qui donne des fruits rouges est associée au feu : le rouge du feu et le rouge du sang s'excluent, leur conjonction provoque un état pathogène caractérisé par la couleur rouge : rougeur de l'ail blessé par une épine ou infecté par la conjonctivite (cf. p. 112). 
périodicité de leurs menstruations à celle de la lune. Ce rapport de similitude est souligné dans la langue : d'une femme indisposée assise sur une bûche on dit a roo (elle est assise) et c'est précisément ce qu'on dit lorsqu'un croissant de lune apparaît à l'ouest. Une femme qui a ses règles s'intègre ainsi aux cycles cosmiques et nous verrons dans un moment quels liens les Indiens établissent entre la lune et le sang.

Jamais la jeune fille ne doit quitter son refuge et paraître aux yeux des autres. Si elle venait à s'exposer aux regards masculins, c'est la communauté tout entière qui serait menacée. Des récits mythiques évoquent ces périls et justifient pleinement que le rituel soit accompli strictement et sans retard. Dans un mythe, une jeune fille indisposée sort la nuit pour participer en compagnie des autres femmes au rituel de chasse heri sur la place centrale; à peine rejoint-elle ses compagnes, à peine sa voix retentit-elle qu'une eau chthonienne surgit du sol qui s'amollit immédiatement ; tout s'y enfonce, les personnes et les choses. Dans un autre récit, une femme quitte subrepticement son abri pour s'en aller rejoindre son époux qui chasse des singes araignées sur un rocher; ils se transforment en pierre. Glissement dans le monde chthonien et pétrification sanctionnent la sortie d'une recluse avant l'achèvement du rituel. Dans un autre mythe, enfin, une jeune fille a la tête coupée par une torsade de coton neuf qu'elle s'était mise trop tôt.

Cet état rituel dure de dix à quinze jours. Lorsqu'il prend fin, la peau est crasseuse et le corps affaibli par le jeûne. On détache alors les «feuilles à menstrues »; l'isolement prend fin ; l'alimentation redevient normale; il n'est pas encore possible de se laver. L'organisme reprend des forces; c'est alors qu'a lieu le rite de la parure, appelé paushimou par les Indiens. Un après-midi la jeune fille part dans la forêt en compagnie de sa mère et de quelques autres femmes. Sa tête est tonsurée au moyen d'un fragment de roseau ; tout le corps (même la tonsure) est enduit de roucou (rouge) et, par dessus ce fond de teint, des lignes noires sont tracées. Dans le trou du lobe de l'oreille on enfile des palmes nouvelles d'un vert encore pâle. La taille est enserrée dans un écheveau de fils de coton blanc (il ne doit surtout pas avoir été rougi au roucou). Un fil de coton est attaché aux bras, aux poignets, aux mollets et aux chevilles. Ainsi parée, la jeune 
fille rentre alors à la maison où règne un air de fête : la voici élevée au rang de femme, elle n'est plus " verte » (ruwë), comme disent les Indiens, mais « mûre" (tate).

Notons au passage, et sans en tirer de conséquences pour le moment, que le même rite paushimou est effectué dans trois situations : lorsque les femmes ont leurs premières règles; dans un cas d'homicide; pendant les rites funéraires au moment où les ossements calcinés sont réduits en poudre pour être versés dans les gourdes où ils seront conservés jusqu'à leur consommation. Les Yanomamë $\ddot{e}^{6}$ disent que le jour ne se lève pas lorsque les femmes ont le vagin sanglant ; les Yanomami centraux affirment que c'est l'inceste qui empêche le jour de se lever. $Y$ aurait-il un lien, et de quelle nature, entre la menstruation des femmes et l'inceste? Je ne sais pas encore répondre à cette question. B. Albert parle du rite yipimou, mais ce verbe ne se rapporte pas à l'accomplissement d'un rituel ; il signifie " avoir ses premieres règles ", ou " produire pour la première fois " en parlant de certains arbres comme, par exemple, l'avocatier ; " être pour la première fois en capacité de procréer » est le sens général de ce lexème et son champ sémantique est étroit. Il doit être clair que l'état rituel dans lequel se trouve une femme qui a ses premières règles se dit unokazi et que l'accomplissement des obligations rituelles est traduit par le verbe unokai-mo- $u^{7}$. B. Albert note bien les conséquences cosmologiques (nuit retardée, surgissement d'une eau chthonienne), les transformations (passage au monde inférieur, pétrification) et les

${ }^{6}$ Tout ce que nous disons ici des Yanomamë provient de la thèse de B. Albert (1985). Cette thèse, qui n'a pas encore été publiée, est un des meilleurs travaux qui aient jamais été consacrés aux Yanomami ; en ce qui concerne le rituel, e'est le plus complet. Je reconnais tout-à-fait la validité du cadre d'interprétation qu'elle met en auvre, ainsi que la valeur heuristique de certains des concepts employés. L'analyse linguistique, en revanche, en constitue le point faible ; il faut dire qu'elle a été tentée à une époque où nos connaissances sur la langue ne permettaient pas encore certaines des interprétations proposées par B. Albert. Les différences qui existent entre les Yanomamë et les Yanomami, sauf insuffisance de notre ethnographie et de nos interprétations, sont généralement attribuables à des variations entre les deux sousgroupes culturels.

${ }^{7}$ En séparant les différents morphèmes. 
conséquences physiologiques (vieillissement prématuré ${ }^{8}$, putréfaction des chairs, déchaussement des dents) qu'entraîne la transgression des obligations rituelles.

L'époux d'une femme recluse suit les mêmes prohibitions qu'elle et se trouve dans le même état rituel, mais il n'est ni isolé, ni dissimulé. Il peut même se déplacer dans la forêt à condition de ne pas mettre les pieds dans l'eau (lui et ses compagnons s'enfonceraient sous terre), ni de récolter du miel ${ }^{9}$. Mais comment se présente le rituel unokai tel qu'il est observé par les homicides ? Qu'est-ce, d'abord, qu'un homicide pour les Indiens Yanomami? Aucun décès n'est naturel, sauf celui des vieillards parvenus à un grand âge. La mort est généralement attribuée à une cause surnaturelle, à un être maléfique, disons à des pouvoirs pathogènes ou à la malveillance des êtres humains. Un homme est meurtrier s'il a tué en usant de la violence et en versant le sang, ou s'il a utilisé des substances maléfiques (hëri) pour donner la mort. B. Albert dit que, chez les Yanomamë, le chaman, lorsqu'il tue par l'intermédiaire des hekura, est considéré comme meurtrier et se soumet au rituel unokai ; ce n'est pas le cas chez les Yanomami où un homme n'est considéré comme meurtrier que lorsqu'il perpétue lui-même un meurtre, qu'il le fasse au moyen d'une arme ou d'une substance magique (comme le font les sorciers $o k a$ ). Mais tous les modes d'agression ont ceci en commun qu'ils sont toujours présentés et interprétés comme des modalités de la « prédation symbolique », comme l'écrit B. Albert : tous sont assimilés à un acte de cannibalisme, une des parties vitales de la victime étant dévorée par l'agresseur homicide, que celui-ci soit un être surnaturel ou un homme.

En plus de ses constituants physiques, un être humain est également formé d'autres principes. Le noreshi est le double animal de la personne, leurs deux destins sont liés, tout ce qui arrive à l'un arrive à l'autre. Le pei mi ámo, littéralement le a centre " de la personne, est une véritable allégorie de la vie. Il est à la fois immatériel et anthropo-

\footnotetext{
${ }^{8}$ Ce trait est propre aux Yanomamë, les deux autres sont communs aux deux souscultures.

${ }^{9}$ Au lieu de miel c'est son propre foie qu'il retirerait et il se transformerait en nid d'abeille.
} 
morphe; par convention nous l'appellerons le "principe vital " ${ }^{10}$. Les êtres surnaturels maléfiques s'en emparent pour le dévorer et l'un des buts de la cure chamanique est de leur livrer combat afin de restituer au malade, pour le guérir, son "principe vital ". La mort, dans ce cas, est assimilée à un acte de cannibalisme dont se rendent coupables des êtres surnaturels. Si la mort est causée par les sorciers $\delta k \tilde{a}$ opérant à l'aide d'une substance maléfique, souvent végétale, on assure que son feu "dévore" le malade et le chaman doit alors l'éteindre, ce qu'il fait souvent en se faisant aider par l'esprit luciole (dont le « feu " est froid).

Lorsque la mort est provoquée par un acte de violence commis par un homme, celui-ci est censé avoir symboliquement mangé sa victime, non pas son «principe vital ", mais sa chair et ses parties molles. Là encore la mort est pensée comme résultant d'un acte de cannibalisme. Dans les dialogues cérémonials, la guerre est assimilée à la chasse et partir en guerre se dit "chercher à assouvir sa faim de viande "; les ennemis que l'on poursuit sont assimiles à des bêtes de chasse, les ennemis dont on est la proie sont des pumas ou des jaguars, des mangeurs de viande crue. Helena Valero raconte dans son récit qu'un homicide lui avait confié avoir vomi la graisse et les cheveux de sa victime. Par ailleurs le principe vital de la victime vient tourmenter le meurtrier, il se met à califourchon sur sa poitrine et l'oppresse. Le rituel unokãimou serait donc destiné à mettre en latence l'organisme du meurtrier embarrassé par des reliefs humains et, à la fois, à apaiser le "principe vital» de la victime. La plupart des prescriptions que suivent les femmes qui ont leurs premières règles sont également suivies par les meurtriers. Il existe néanmoins des différences significatives: le meurtrier suspend son hamac entre deux poteaux écorcés plantés en avant des feux, vers la place centrale (au lieu de s'installer à la base du toit, en arrière des feux); il n'est donc pas dissimulé. Le régime alimentaire est également plus strict, il ne mange que la petite espece de banane-plantain $\left(\right.$ kurat $\left.^{h} a\right)$; la grosse (pareami)

${ }^{10}$ Le * principe vital * dit pei mi âmo pour les vivants se détache du corps à la mort et prend alors le nom de pei no uhutipi. Par ailleurs, le pei mi âmo (le centre) s'oppose au pei siki (l'enveloppe) comme la substance s'oppose à la forme. 
lui donnerait des démangeaisons ; il ne doit surtout pas manger de rasha sinon ses yeux seraient rongés par la conjonctivite. Plusieurs fois il doit se frotter le corps avec les "feuilles à menstrues". Tous les meurtriers sont faibles, malades ${ }^{11}$, ils prétendent que dans cet état leur haleine exhale une odeur de charogne ; leurs levres se dessèchent et durcissent ; leur peau peut se craqueler, mais elle est huileuse, surtout au front ${ }^{12}$; leurs yeux se brouillent et sont comme enflés; ils sont menacés de pourriture si le rituel n'est pas bien accompli. Surtout, le principe vital (pei mi ámo ou pei no uhutipi) de leurs victimes les oppresse et peut mettre leurs jours en péril. Lorsque le rituel est mal suivi, si le meurtrier mange trop ou consomme des aliments interdits, s'il omet de se gratter avec un bâton épointé, ou s'il commet quelque autre acte prohibe, le pei no uhutipi le rendra malade et il se mettra à gonfler ${ }^{13}$.

Une question se pose alors : le même rituel est-il imposé parce que les deux situations relèvent d'un même traitement symbolique? Est-ce bien le sang qui est l'élément commun ? Sang versé par les femmes lors de leurs premières menstrues, sang que versent les homicides ? Mais les sorciers $\delta k a ̊$ qui se soumettent également au rite unokăi n'ont pas versé de sang : ils ont fait périr leurs victimes en projetant sur elles une substance réputée mortelle, qui agit sur l'organisme sans aucun épanchement de sang; nous avons dit que le mal, qui peut être mortel, provenait d'un feu interne dévorant : il est

11 On parle de la fin du rituel comme d'une guérison (haro-).

12 On dit que c'est la graisse du mort qui s'écoule ainsi.

${ }^{13}$ Le pei no uhutipi de certains êtres peut être particulièrement dangereux. Celui d'une certaine espèce de tortue doit être tué magiquement par les chamans pour que la bête puisse être mangée ; celui des cufs de l'oiseau hõrãma (un tinamidé de l'espèce Crypturellus associé au déluge, aux pécaris et au souffle des Hommes) doit être neutralisé à l'aide d'une pointe de flèche. Le pei no uhutipi des victimes est redoutable pour les meurtriers et le rite est aussi censé les protéger : s'ils le suivent mal ils gonflent (hihatho-) ou pourrissent (washë-) (le gonflement du corps est aussi une annonce de la pourriture). Dans un mythe une tortue est tuée par le pei no uhutipi du jaguar qu'elle vient d'égorger. 
accompagné de fièvre ${ }^{14}$. Il semble bien plutôt que ces deux situations soient vécues comme des transitions périlleuses, des moments de grande impureté accompagnant des transformations ou des mutations, et c'est la raison pour laquelle le même traitement rituel leur est appliqué. Mais si, dans le cas des femmes qui ont leurs premières menstrues - et dans celui de leur époux, contaminé -, c'est bien le sang vaginal qui est l'élément impur et dangereux, dans le cas des meurtriers, cannibales, c'est la pourriture, la chair en décomposition, qui menace : les meurtriers sont malades de l'acte d'anthropophagie dont ils se sont rendus coupables, le pei no uhutipi de leur victime les affecte et les tourmente. Dans tous les cas, le rituel unokaimou accompagne une transition, coïncide avec un état d'impureté, protège des organismes affaiblis.

B. Albert traduit unokäi par : " état d'homicide ". Nous comprenons bien que cette interprétation n'est pas entièrement recevable puisque cette situation rituelle est aussi celle des femmes qui ont leurs premieres règles, et celle de leurs époux. B. Albert part de l'analyse du terme rãakai, "être malade ", qu'il considère comme un verbe ; il le décompose de la manière suivante: rãa , "être malade ${ }^{15}$ et "käi ", " avec, accompagné de ". Puis il propose une série d'exemples où il est évident que rãa est le lexème "malade [+ état] " et que kãi est une particule autonome et invariable qui, dans la syntaxe yanomami, signifie qu'une chose s'accomplit dans un état déterminé, ou en même temps qu'une autre. Il s'ensuit que la particule kãi ne saurait en aucune manière être suffixée à rãa. B. Albert dit aussi que

${ }^{14} \mathrm{~L}$ 'usage de la sorcellerie et des substances magiques pour tuer s'insère également dans une logique de vengeance et de guerre. Très souvent les guerres sont déclenchées par un décès attribué à ces pratiques qui exigent la vengeance, tout comme un meurtre provoqué par une agression corporelle et l'usage d'une arme (épanchement de sang). Lorsqu'un conflit est ouvert, la guerre peut être conduite en utilisant indifféremment l'un ou l'autre moyen. Mais, pour qu'une communauté accuse une autre d'avoir utilisé la sorcellerie pour tuer l'un des siens, il faut que les relations soient déjà dégradées entre elles, ou que des traces nettes indiquent indiscutablement le coupable.

15 Chez. les Yanomami rãã n'a pas ce sens; ce mot signifie * être éveillé * et rãâmou a le sens de * gémir * ; être malade se dit de différentes manières ; les deux mots les plus usuels sont hariri (état) et pëi (procès). 
le mot wayuwayu $u^{16}$ est un synonyme de raa ; mais wayuwayu est un substantif que l'on pourrait traduire, s'il le fallait vraiment, par « démon de la maladie ». Enfin, en réfléchissant sur l'étymologie de unokai, B. Albert fait l'hypothèse que ce terme est formé sur le modele lexical de rãakai, interprétation qui, nous venons de le voir, n'est pas recevable. Décomposant ensuite unokai en plusieurs morphèmes, B. Albert affirme alors que uno a le sens de "trace ", de * marque " et que kãi a celui de « avec, accompagné de ». Répétons à nouveau que, dans une chaîne syntaxique, kãi est une particule libre et invariable qui sert à l'articulation de l'énoncé ; en aucun cas elle ne peut entrer dans la formation d'un lexeme ; il n'est pas non plus possible de lui suffixer la désinence -mo-, comme c'est le cas dans unoká-mo-u. Nous pourrions poursuivre dans le même sens en discutant le sens possible des prétendus morphèmes * $u$ " et « no " de $u$-no-kai dans l'interprétation sémantique qu'en donne B. Albert. Il est préférable de considérer unokäi comme un lexème indécomposable dont unokái-mo-u est le dérivé; le sens du morphème -mo- étant [+ voie moyenne] dans le cas présent (le - $u$ final est une désinence temporelle, il indique le présent progressif).

En ce qui concerne le "principe vital " l'interprétation des Yanomamë analysée par B. Albert differe aussi de celle des Yanomami centraux qui considerent le " principe vital " comme un " centre ", une substance immatérielle, une énergie dont l'existence peut être, après la mort, indépendante du corps ; c'est un peu comme une flamme ou, comme le suggère le mot no uhutipi, un reflet, qui persisterait provisoirement après lui et se souviendrait de ses origines. Pour les Yanomamë, le «principe vital » est étroitement associé au sang et manger le «principe vital " d'une personne en la tuant, c'est ingérer son sang. Nous avons bien vu que les Yanomami ne disaient pas de manger le principe vital de leurs victimes, mais leurs corps matériel,

${ }^{16}$ Les Yanomami centraux disent wayuwayu $k e ̈ ~ k i$, au pluriel, ou, plus fréquemment, shawara $k \ddot{e} k i$. Les deux mots sont synonymes dans tous les contextes ; ils désignent des êtres surnaturels responsables de certaines maladies et des épidémies. 
et que, justement, c'est le principe vital qui, sous le nom de pei no uhutipi, venait les tourmenter ensuite.

La femme qui a ses menstrues et le meurtrier cannibale sont l'un et l'autre impurs, en dehors de la culture, et c'est la raison pour laquelle les prohibitions et les obligations qui accompagnent le rituel unokai peuvent glisser indifféremment en deçà ou au delà des normes culturelles usuelles, l'important étant qu'elles soient hors culture. Le rite exige aussi que les fonctions physiologiques soient mises en veilleuse et la communication abolie ; le corps est immobilisé, les liens sociaux sont temporairement coupés. Le rite paushimou de la parure qui suit et clôture le rite unokaimou est un rite de purification finale (par l'eau, les colorants et les parfums), de régénérescence et de réinsertion sociale; les hamacs des meurtriers sont alors suspendus dans les arbres, on y attache les petits grattoirs en bois et le tube à boire $^{17}$. Le rite unokäi ne serait-il pas ce que Van Gennep a appelé un rite de passage ? La femme, lorsqu'elle a ses premières règles, de " verte " qu'elle était, devient " mûre ", jadis stérile elle accède à l'état de procréatrice périodiquement fertile. Souvenons-nous également que de nombreuses sociétés amérindiennes exigeaient la réclusion des meurtriers ; ceux-ci «mouraient », en quelque sorte, pour renaître ensuite et changer de nom : ceci pour échapper aux esprits vindicatifs de leurs victimes. Et puis, de la même manière que la jeune fille qui a ses premières règles est transformée et change de statut, le meurtre transforme le meurtrier : dans la poitrine de tout homicide vient se loger un "démon de la guerre" (åiãmo). Les aiămori forment un peuple de cruelles créatures surnaturelles ; immortels ils se livrent une guerre incessante ; blessés ils lèchent le sang qui s'écoule de leur plaie. Le pei no uhutipi d'un de ces êtres peut venir se loger dans un homme pour le posséder et le tourmenter. Par extension le mot áämo peut désigner une personne courageuse et stoïque (et être synonyme de wait ${ }^{h}$ eri dans ce contexte-la), voire un objet pour tuer. D'un guerrier on dit que sa poitrine, littéralement, "vaut un démon de la guerre " (pariki no aiamopi).

17 On dit que ce lieu est hanté par les spectres des victimes. 
Au terme de cette analyse du sens qu'ont le sang et le rituel unoka $i$ chez les Yanomami, nous voudrions relier ce qui vient d'être dit cles meurtriers à leur statut social et à des phénomènes sociologiques fondamentaux. En effet tout le système rituel yanomami est couplé et, pour ainsi dire, amalgamé, au système de réciprocité et de communication; les différents types de périodicité (cosmique, biologique et sociale) se trouvent dans un étroit rapport de corrélation : la pensée indigène les conçoit globalement dans leur interdépendance.

Dans les temps primordiaux régnait un jour sans fin, le soleil demeurait au zénith, les hommes ne savaient pas ce qu'étaient le crépuscule, la nuit. On chassait ; les enfants jouaient ; on dormait n'importe quand; on faisait l'amour à la vue de tous ; c'était une époque sans querelle et sans guerre, la paix régnait. Tout aurait été pour le mieux si l'on n'avait entendu, proche et lancinant, le chant plaintif de Titiri nommant les montagnes, les collines, les rochers, les cours d'eau. Cette plainte ininterrompue terrifiait les ancêtres. Tout autour de l'endroit où perchait le démon régnait l'obscurité la plus totale. Lassés, les ancêtres décidèrent un jour de tuer l'oiseau démoniaque ; ils allumerent un grand feu pour éclairer leur cible et lui donnèrent la mort. Alors la nuit s'étendit. Des êtres surnaturels ouvrirent la poitrine de Titiri et de son sang naquirent les esprits du crépuscule et du matin, les weyari/harikari. Depuis lors la nuit et le jour se succèdent. Cheminant de l'orient vers l'occident, Scorpion passe le premier à midi et, pendant qu'il chemine, il tue toutes les bêtes qu’il rencontre. Les esprits du matin et du crépuscule passent ensuite, ils précèdent de peu le crépuscule ou l'aube. Ils ramassent et mangent les animaux tués par Scorpion, en choisissant de préférence les paresseux les plus coriaces (pour les Yanomami, les paresseux sont des hommes qui se sont métamorphosés à la suite d'un inceste sororal). Les esprits du matin et du crépuscule sont aussitôt suivis de Titiri, lorsque tombe la nuit ou que le jour se leve. Pendant la nuit, mais en sens inverse cette fois, c'est à dire de l'occident vers l'orient, ces êtres accomplissent le même trajet. Le précurseur de Titiri, le démon de la nuit, c'est le mutum (Crax nigra). La chair de cet oiseau est une de celles que préferent les Yanomami ; ses plumes servent à fabriquer les 
empennes des fleches et entrent dans la parure. Le plumage du mutum est très contrasté : d'un noir sombre sur tout le corps, il est d'un blanc éclatant sur le ventre. Ce contraste violent du blanc et du noir est particulièrement approprié pour symboliser l'opposition du jour et de la nuit. Le mutum est aussi lié à une autre périodicité, celle de la saison sèche et de la saison humide qui, chez les Yanomami, se succedent d'octobre à mars et d'avril à septembre. En effet, pendant la saison sèche, le mâle, vers deux ou trois heures du matin, se met à chanter, à "pleurer " disent les Indiens. A cette époque ces oiseaux sont un gibier de choix. Les chasseurs partent la nuit dès qu'ils supposent que les mutums chantent ; ils s'éclairent en agitant devant eux une poignée de tisons. Lorsqu'ils entendent le chant de l'oiseau, ils se guident sur lui pour approcher le plus pres possible ; quand le jour se lève ils décochent à l'oiseau leurs flèches.

Le démon de la nuit, Titiri, est associé au mutum. L'oiseau démon du mythe était un mutum, il " pleurait ", comme lui. Le démon de la nuit appartient à l'une des deux classes d'êtres surnaturels reconnues par les Yanomami ; il est anthropomorphe ; ses cheveux sont blancs, et frisés comme les plumes de la crête du mutum. Titiri possède un énorme pénis; il s'accouple avec les humains à leur insu pendant qu'ils dorment, il déchire leurs chairs avec son membre et s'empare de leur "principe vital". Le chemin que suit Titiri est couvert d'épines qui lui blessent les pieds et le démon clopine. D'une certaine manière, on peut dire que Titiri perfore avec son pénis en même temps qu'il est perforé par les épines. Scorpion est aussi un personnage de la mythologie. C'est le symbole du chasseur infaillible. Un jour un grand chaman mourut, il se transforma en un être surnaturel appelé Lune (au masculin en yanomami). Durant la nuit qui suivit son décès, Lune vint dévorer les os de son propre cadavre qui venait d'être incineré ; surpris par son propre fils alors qu'il achevait son macabre festin, Lune s'éleva au ciel pendant que les hommes lui décochaient des flèches qui, toutes, manquèrent leur cible. Au moment où Lune allait disparaître derrière les nuages, Scorpion se leva de son hamac, ajusta sa fleche, tira et atteignit Lune. Son sang goutta sur la terre et chaque goutte de sang donna naissance à des hommes armés qui se combattirent aussitôt. La guerre venait de naître. 
L'interprétation complète de l'ensemble que nous venons de présenter nous conduirait trop loin et nous obligerait à exploiter un matériel ethnographique de plus en plus important. Mais le problème du sang oblige à poser celui de la nature de la périodicité des cycles naturels et biologiques, de l'échange et de la réciprocité, des relations pacifiques et de la guerre. Poursuivons donc un peu notre apparente digression et abordons brievement la question du dialogue cérémonial : c'est fondamentalement un échange de mots qui lie deux à deux et pendant toute la nuit des partenaires issus de deux ou plusieurs communautés, les uns étant des visiteurs, les autres des amphitryons. Il sert a maintenir des relations pacifiques ou à les restaurer lorsqu'elles se degradent; il appartient à un vaste systeme de communication et s'insère dans la série des échanges pacifiques. Le mythe d'origine de la nuit que nous avons évoqué est aussi, au moins implicitement, un mythe d'origine du dialogue cérémonial. Celui-ci est nocturne, il commence au crépuscule, lorsque Titiri vient juste de passer, et se termine à l'aube, lorsque les étoiles s'estompent à l'orient, au moment où passe à nouveau le « démon » de la nuit. Il existe une relation directe entre la périodicité du jour et de la nuit et celle des échanges. Mais pourquoi la plainte de Titiri effrayait-elle tant les ancêtres? Parce que cette voix était solitaire (une caricature du dialogue), l'espace qu'elle s'évertuait obstinément à nommer sans fin n'était ni orienté ni même inséré dans le temps ; et puis, surtout, cet espace était vide, sans groupes humains, jamais Titiri ne nomme aucune communauté. Sa mort brutale provoque non seulement la périodicité quotidienne - le jour et la nuit se succèdent -, elle instaure également un espace orienté : dorénavant le soleil ne reste pas fixé au zénith, il se lève à l'orient et se couche à l'occident. Est et Ouest constituent un axe le long duquel se déplacent Scorpion, le chasseur, les démons de l'aube et du crépuscule, friands de paresseux coriaces, Titiri le clopinant enfin. A la différence de la plainte de Titiri, le dialogue cérémonial ne se contente pas de nommer les lieux, il peuple l'espace, il y place des communautés, il le socialise et l'humanise; mais il fait plus encore puisque le monde surnaturel, réplique idéalisée du monde des hommes, y trouve également place : 
les montagnes et les rochers sont habités par des hekura ${ }^{18}$. L'univers s'y loge même à travers un système complexe de représentations. A propos des mythes d'origine du jour et de la nuit, Lévi-Strauss écrit qu'ils «conceptualisent en même temps l'espace et le temps". L'originalité des Yanomami est peut-être d'y parvenir par l'intermédiaire de la nomination et de l'échange.

D'un point de vue strictement métaphorique il n'est pas excessif de dire que Scorpion nourrit de bêtes sauvages la périodicité du jour et de la nuit, il le fait même de préférence avec des incestueux métamorphosés en paresseux. Or les Yanomami centraux affirment qu'un inceste sororal empêche le jour de se lever. Le mythe d'origine de la nuit et celui où l'on parle de la naissance d'un peuple de guerriers, de la guerre donc, se complètent : l'un instaure la succession du jour et de la nuit, l'autre la périodicité des cycles lunaire et biologique. Les femmes mettent clairement en relation la périodicité des cycles menstruels avec celle de la lune. "Lune " est ainsi doublement associé au sang: la régularité de ses apparitions est couplée à la périodicité des flux menstruels, son sang est aussi celui de la guerre puisqu'il crée la violence et que celle-ci débouche sur la mort. Le temps cyclique, c'est aussi la procréation et la mort. C'est aux mêmes conclusions qu'arrive Lévi-Strauss lorsqu'il associe la périodicité mensuelle à la procréation et à la mort. Lorsqu'il est surpris, Lune vient de consommer ses propres cendres, les restes osseux de son cadavre incinéré. Le scandale est double : ce faisant, non seulement Lune commet un acte d'auto-cannibalisme (il se mange lui-même), mais il rend tout échange impossible, ses cendres sont soustraites à son jeu, et l'on se demande si finalement la pensée indigène n'assimile pas à un inceste cet acte, répugnant pour elle. Rappelons que les cendres des morts sont distribuées entre les cognats et les alliés et que leur consommation donne lieu à des cérémonies accompagnées de festins. La poudre osseuse est partagée et ingérée, on se l'échange au cours de certains rituels : les morts entrent dans le

18 Les hekura sont des êtres sumaturels associés à des éléments naturels tels que les plantes, les animaux, les montagnes, le vent, etc... Domestiqués, ils viennent loger dans la poitrine des chamanes et leur donnent du pouvoir. 
cycle des échanges et de la réciprocite ${ }^{19}$. Ces fêtes sont elles-mêmes accompagnées de dons de nourriture et de tabac; des dialogues cérémonials y prennent place; des échanges commerciaux les clôturent. Il faut enfin signaler, même si cela semble un peu trop évident, que, tout comme les homicides, les femmes sont porteuses de mort justement parce qu'elles donnent la vie : tout être biologique est porteur de sa propre fin, toute naissance implique une mort future. Il faut aussi ajouter que ce sont les femmes qui, se noircissant les pommettes des joues, portent le deuil lors d'un décès, et que les hommes se noircissent la peau lorsqu'ils partent en guerre. Le noir symbolise à la fois la nuit, la guerre et la mort.

Pour B. Albert, tout comme le corps du meurtrier, celui de la jeune fille qui a ses premières règles est envahi par les effets d'une naturalité excessive qui se trouve dans un premier temps enregistrée et transposée dans le rite sur le plan social et culturel. Cette naturalité est ensuite symboliquement circonscrite, et cuite : d'où la nécessité d'un feu abondant et l'emploi d'instruments inhabituels (grattoir, tube à boire). Cette cuisson est parachevée lors de la clôture du rite par une reculturation graduelle qui débute avec la mise en place de la parure de la jeune fille et sa réinsertion progressive dans les activités.

A propos de la périodicité, B. Albert ajoute une dimension nouvelle. Pour lui, en effet, le rite de puberté (féminin) et le rite d'homicide (masculin) forment en quelque sorte deux des côtés d'un « triangle culturel " de la périodicité :

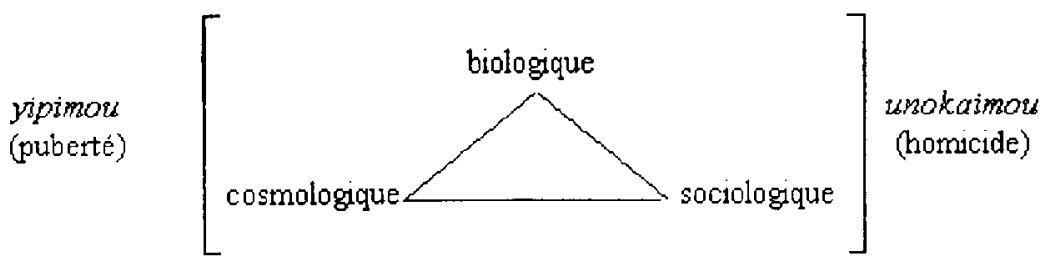

19 La consommation des cendres de la victime d'un meurtre précède toujours l'expédition guerrière destinée à la venger. 
Le troisième côté de ce triangle, ajoute B. Albert, devrait être occupé par un rite dont le travail symbolique assurerait une maîtrise culturelle simultanée de la périodicité cosmologique et de la périodicité sociologique. B. Albert pense que c'est précisément la place qu'occupe le rituel funéraire. Nous avons pourtant vu que unokãi ne pouvait pas être traduit exactement par homicide, nous avons vu également que ce terme s'appliquait à la fois à la situation rituelle d'une jeune fille ayant ses premières règles et à celle de quelqu'un qui a donné la mort, avec ou sans épanchement de sang, à celle, enfin, de l'époux d'une jeune recluse (probablement contaminé par l'état d'impureté dans lequel se trouve sa femme).

Je dois confesser qu'arrivé à ce point je n'ai plus grand chose à dire sur le probleme du sang chez les Yanomami. Il faut pourtant prolonger cet exposé afin d'aboutir à une vision plus globale qui montre, au moins dans ses grandes lignes, de quelle manière l'homicide, le guerrier, s'incorpore, sous l'effet de l'échange et de la réciprocité, dans un vaste systeme de communication. Il ne suffit pas de parler du statut rituel de l'homicide : quel est, sur un plan plus général, le statut du guerrier, quel rôle assume-t-il dans la société ? Il faut bien sûr évaluer les aspects sociologiques de l'homicide et de l'agression en général, et voir comment ceux-ci s'intègrent dans le système global de communication.

Le dialogue cérémonial est un échange pacifique qui porte sur des mots ; mais les échanges se poursuivent même dans les relations d'hostilité : on échange alors des coups, des blessures, des cadavres. Chez les Yanomami le système de communication est régi par la stricte obligation de la réciprocité, dans tous les domaines de l'activité sociale et de la vie rituelle; ce système de relations complexes constitue le domaine du politique et celui-ci parcourt toute la société. Que dit là-dessus la théorie de la réciprocité ? Pour Mauss, c'est l'obligation de donner et de recevoir qui constitue le fondement des relations pacifiques. L'échange est le commun dénominateur d'un grand nombre d'activités sociales hétérogènes. Commentant l'Essai sur le don, Lévi-Strauss écrit que : " l'échange constitue le phénomène premier et que les différents produits de l'activité sociale (ustensiles, produits 
manufacturés, aliments, formules magiques, ornements, danses, chants et mythes) sont comparables pour posséder en commun le caractère d'être transférables et substituables ». De son côté Sahlins nous met en garde contre l'erreur qui consisterait à interpréter l'échange en des termes exclusivement matériels ; il s'agit bien, en effet, d'un acte social.

$\mathrm{Au}$ sein du groupe familial règne ce que Sahlins nomme " la réciprocité généralisée "; au-delà du cercle familial la réciprocité équilibrée régit l'échange ; et c'est dans ses rapports avec les ennemis et les étrangers (autres Indiens, créoles et "Blancs ") que la réciprocité devient négative, ou plutôt elle disparaît totalement puisqu'il s'agit de prendre (en obtenant par la force ou la menace, ou en volant) sans jamais rien donner en échange. Entre les membres d'une même famille et au sein de la faction, ce sont les échanges de nourriture et de services qui prédominent ; les règles de distribution du gibier sont très strictes et le chasseur ne doit pas consommer les animaux qu'il tue, il doit les donner ou les partager sous peine de devenir sina, mauvais tireur, mauvais pisteur. Un ou plusieurs groupes familiaux ${ }^{20}$, une faction ou une communauté toute entière peuvent toujours se faire inviter, héberger et alimenter au cours d'une visite, ou lorsque qu'il n'y a rien à récolter dans les plantations. Il est toujours possible de solliciter l'aide d'un parent en période de rareté, lorsque la nourriture vient à manquer. Le vocabulaire consacré à la réciprocité des dons d'aliments est tout à fait expressif : shoayou, se faire héberger et alimenter réciproquement (le suffixe -yo est la marque du réciproque) ; ni $t^{h} a o$, offrir de la nourriture à un visiteur ; kahiki no koamai, rendre un don de nourriture, littéralement « restituer la valeur de la bouche »; nowã ohiamou, «demander à être hébergé et nourri en période de disette ". Les biens s'échangent généralement entre des personnes appartenant à des communautés distinctes, très souvent à l'occasion de visites, et l'échange commercial avec des partenaires est le but de ces visites. Tout objet donné ou reçu possède son « pedigree ", son histoire

20 Une famille est généralement composée d'un couple et de ses enfants ; quelquefois le père ou la mère de l'un ou l'autre des époux s'ajoute à ce groupe. 
particuliere ; il a aussi une marque distinctive (imi no) qui est celle du premier donateur : "la trace du doigt". Deux partenaires commerciaux disent qu'ils se " possèdent mutuellement le bras " (poko $t^{h}$ apoyou). Les fêtes et les cérémonies sont autant d'occasions d'échanges ; pendant les séances de chamanisme les officiants s'offrent mutuellement des « esprits " (hekura).

La série des rites funéraires est une splendide et dramatique illustration de ces principes. Lorsque le cadavre a été brûlé et réduit à des os calcinés, ces os sont soigneusement ramassés et conservés dans un panier pendant une semaine environ. Alors ils sont pilés dans un mortier et réduits en une poudre très fine au cours d'une cérémonie appelée paushimou, cette poudre est versée dans des gourdes fermées à la cire et distribuées entre différents parents. Plus tard ces cendres seront consommées (offertes à des consommateurs) dans une soupe de banane. Chaque cérémonie, l'incinération du cadavre exceptée, donne lieu à des festins et à des distributions de viande (des prestations) lors des inévitables chasses de longue duree, mais, surtout, c'est le corps même du défunt qui est objet d'échange et de consommation.

Si nous explorons un moment le code moral yanomami, nous y découvrons d'autres convergences. Il s'organise en gros autour de deux vertus dominantes, et complémentaires : la générosité et le courage. $\mathrm{Ce}$ n'est pas par hasard. La générosité suppose des dons fréquents, le courage comporte l'obligation de se venger, de rendre coup pour coup, cadavre pour cadavre. Il semble bien que la relation établie entre la générosité et le courage provienne d'une analogie très étroite : ces deux vertus reposent sur la logique du don et du contre don, de l'échange réciproque ; l'une cependant est du domaine des relations pacifiques, l'autre règle la violence et la guerre. C'est tout à fait ce que disent les Yanomami, pour eux la générosité est nécessairement associée au courage : un homme courageux est toujours généreux ; l'homme idéal s'accomplit dans l'échange : dans son commerce avec les autres, dans la paix et dans la guerre, il doit savoir pleinement participer à toutes les formes d'échange. La langue est elle-même marquée puisque la réciprocité est grammaticalisée et le morphème -yo est, dans les verbes, la marque du réciproque ; le partage, aussi, est marqué grammaticalement et tous les verbes qui peuvent avoir le sens 
général de « agir sur un objet pour le partager et/ou l'offrir " possèdent des désinences particulières : certains suffixes indiquent si l'on ne préleve qu'un morceau ou si l'on coupe en deux ou en plusieurs parts, d'autres si c'est pour donner, s'approprier, ou si l'on reçoit ou pense recevoir.

Si l'on passe du niveau du morphème et de la désinence verbale à celui des mots et des expressions, on se rend aussitôt compte que les différentes modalités de l'échange, et la nature des objets qui s'échangent, sont constamment confondues et que ces objets, matériels ou immatériels, sont bien * interchangeables ". Voici quelques illustrations. no koamat signifie habituellement " assurer la réciprocité d'un échange (commercial) " c'est, littéralement, "restituer la marque ", c'est à dire la * valeur "; dans un certain contexte cette locution peut avoir aussi le sens de " accomplir la vengeance de sang ». Le lexème matohi peut vouloir dire : « objet pour l'échange », " bien personnel ", " restes d'un mort après l'incinération ", " gourde funéraire ", « objet ou substance servant à tuer ". rahaka toayou peut tout aussi bien signifier "échanger mutuellement des pointes lancéolées " (au cours d'un échange commercial pacifique) que " se faire la guerre ". hiima toayou, " échanger des chiens ", peut aussi être traduit par " tuer pour venger un mort " et atari shetekeprat, littéralement " partager en deux une pointe-harpon ", signifie en réalité * assumer la vengeance de sang (en tuant quelqu'un) ", c'est la métaphore du partage qui, cette fois, figure la vengeance ; atari ahi, la pointe-harpon, peut avoir le sens d'homme dans de nombreuses expressions. Dans l'exemple ici donné, chaque partie du conflit se partage une moitié de pointe, chacune prend un mort.

Poursuivons encore un instant notre promenade à travers les mots. Etre en guerre se dit habituellement niyayou en yanomami : « se tirer mutuellement (des fleches)». Stricto sensu, le mot guerre n'existe pas, mais l'idée peut s'exprimer de différentes façons qui nous introduisent toutes au cour des conceptions indigènes: na wayou, "se faire l'amour "; totihitayou, "se faire mutuellement jouir »; ohëmayou, "s'affamer l'un l'autre" ; yahatuayou, "s'insulter mutuellement (en dénonçant les imperfections physiques de son rival)"; wäha wayoayou, "se raconter des histoires». A travers ces différentes 
expressions on découvre que certains types de relations sont parfaitement interchangeables : un échange guerrier peut prendre la place d'un échange pacifique, différents codes (alimentaire, sexuel, verbal) peuvent permuter, les objets de l'échange étant eux-mêmes variables. C'est la manière qu'ont les Indiens de percevoir la réalité qui nous est ainsi révélée, leur manière de la penser. Mais, répétons-le, l'analyse sémantique nous confirme que l'échange réciproque couvre à la fois le champ des relations pacifiques et celles de la violence et de la guerre. Cette conceptualisation se concrétise dans des préceptes moraux et une pratique: l'échange doit être réciproque, le don doit être rendu, les coups et les morts aussi ; il faut à la fois être vaillant et généreux. Cela peut s'exprimer d'une autre manière : nos concepts de paix et de guerre n'ont aucune réalité dans le système conceptuel yanomami; ces relations nous apparaissent antagonistes, les Indiens les voient plutôt comme complémentaires, et de même nature. Les relations pacifiques et l'hostilité sont l'une et l'autre l'expression de la structure sociale, elles sont perçues comme deux modalités de l'échange et de la réciprocité. Le système politique yanomami peut être comparé à une énorme machine à communiquer mue par l'obligation de l'échange réciproque, les différents codes utilisés et les objets qui circulent sont bien substituables. Brocardant un peu Clausewitz, on pourrait dire que la guerre est la poursuite de l'échange par d'autres moyens, et non pas exactement, comme l'a pensé Lévi-Strauss ${ }^{21}$, une transaction commerciale qui aurait mal tournée.

Jacques Lizot

C.N.R.S.

21 Dans un article ancien qu'il n'a d'ailleurs jamais republié, un peu comme s'il renonçait à l'interprétation que je viens de mentionner. 


\section{Références bibliographiques}

Albert, Bruce

1985 Temps du sang, temps des cendres : représentation de la maladie, système rituel et espace politique chez les Yanomami du Sud Est. Paris, Université de Paris X-Nanterre, Thèse de Doctorat.

Lévi-Strauss, Claude

1945 * Guerre et commerce chez les Indiens de l'Amérique du Sud *, Renaissance, vol. 1, New York.

1964 Le cru et le cuit, Paris, Plon.

1966 * Introduction à l'ouvre de Marcel Mauss * in Sociologie et Anthropologie, Marcel Mauss, IX-LII, Paris, Presses Universitaires de France.

1968 L'Origine des manières de table, Paris, Plon.

1971 L'Homme nu, Paris, Plon.

Lizot, Jacques

1976 Le Cercle des feux, Paris, Le Seuil.

1989a No patapi tëhë - En tiempos de los antepasados, Puerto Ayacucho, Vicariato Apostólico.

1989 b * A propos de la guerre : une réponse à N. A. Chagnon *, Journal de la Société des Américanistes, LXXV, 91-113.

1994 *Words in the Night : The Ceremonial Dialogue - One Expression of Peaceful Relationship Among the Yanomami », in The Anthropology of Peace and Nonviolence, Leslie E. Sponsel \& Thomas Gregor éds, London, Lynne Rienner Publishers, 213-240.

Mauss, Marcel

1966 Sociologie et Anthropologie, Paris, Presses Universitaires de France, (1ère ed. 1923).

Sahlins, Marshall

1972 Stone Age Economics, Chicago, Aldine.

Valero, Helena

1984 Yo soy napëyoma. Relato de una mujer raptada por los indígenas Yanomami, Caracas, Fundación La Salle de Ciencias Naturales. 